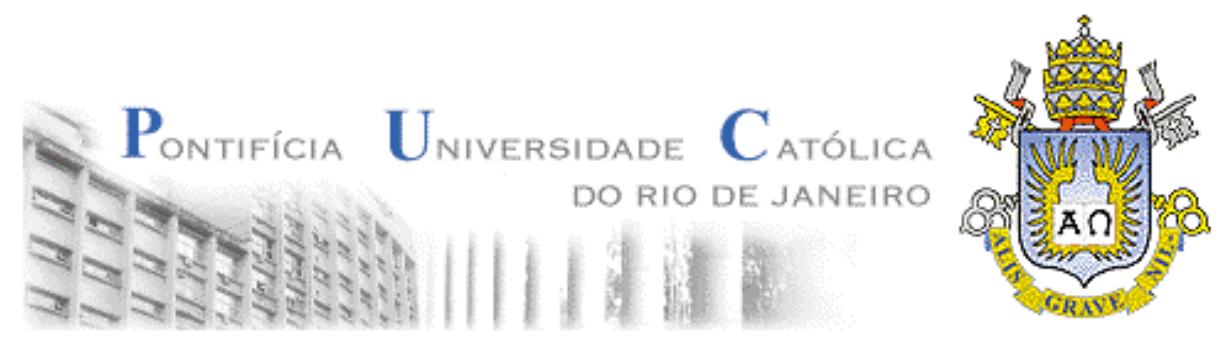

Eduardo Pasquetti

\title{
Estabilidade Estática e Dinâmica de Torres Estaiadas
}

\begin{abstract}
Dissertação de Mestrado
Dissertação apresentada como requisito parcial para obtenção do título de Mestre pelo Programa de PósGraduação em Engenharia Civil da PUC-Rio. Área de concentração: Estruturas.
\end{abstract}

Orientador: Paulo Batista Gonçalves 


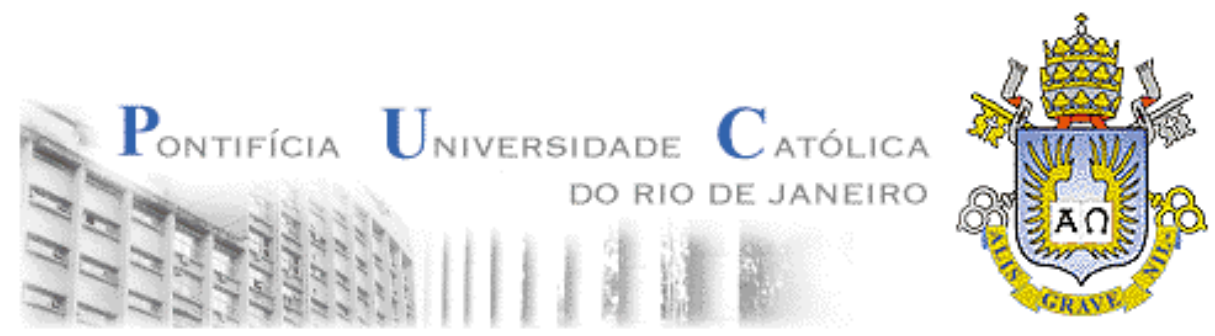

Eduardo Pasquetti

\section{Estabilidade Estática e Dinâmica de Torres Estaiadas}

Dissertação apresentada como requisito parcial para obtenção do título de Mestre pelo Programa de PósGraduação em Engenharia Civil da PUC-Rio. Aprovada pela Comissão Examinadora abaixo assinada.

\section{Paulo Batista Gonçalves \\ Orientador PUC-Rio}

\section{João Luis Pascal Roehl} PUC-Rio

\section{Raul Rosas e Silva PUC-Rio}

Zenón José G. Nuñez del Prado Universidade Federal de Goiás

Ney Augusto Dumont Coordenador Setorial do Centro Técnico Científico - PUC-Rio 
Todos os direitos reservados. É proibida a reprodução total ou parcial do trabalho sem autorização da universidade, do autor e do orientador.

\section{Eduardo Pasquetti}

Graduou-se Engenheiro Civil em Janeiro de 2001, pela Universidade de Passo Fundo (UPF)

Ficha Catalográfica

Pasquetti, Eduardo
Estabilidade estática e dinâmica de torres
estaiadas / Eduardo Pasquetti; orientador: Paulo Batista
Gonçalvez. - Rio de Janeiro : PUC, Departamento de
Engenharia Civil, 2003.
[18], 81 f. ; 29,7 cm
Dissertação (mestrado) - Pontifícia Universidade
Católica do Rio de Janeiro, Departamento de Engenharia
Civil. $\quad$ Inclui referências bibliográficas.
1. Engenharia civil - Teses. 2. Instabilidade. 3.
Torres estaiadas. 4. Cabos. 5. Dinâmica não-linear. I.
Gonçalvez, Paulo Batista. II. Pontifícia Universidade
Católica do Rio de Janeiro. III. Título.


Aos meus familiares e a todos que contribuíram na realização deste trabalho. 


\section{Agradecimentos}

A PUC-Rio e aos professores do departamento, pela oportunidade.

Ao meu orientador, pela convivência, disponibilidade, incentivo, paciência, pelos livros e conhecimentos transmitidos durante este último ano.

Aos meus professores da graduação: Agenor D. de Meira Jr., Ignacio Iturrioz, Mario Paluch, Moacir Kripka e Zacarias M. Chamberlain, pelo incentivo.

A banca examinadora.

Aos meus colegas durante estes dois anos, de forma especial a Andre L. Müller, Chan Kou Wha, Ricardo A. Chaves, Sandoval J. Rodrigues Jr. e Walter M. Guimarães Jr., pela convivência e troca de conhecimento.

Aos funcionários do departamento.

A CNPQ e a FAPERJ, pelo auxílio financeiro. 


\section{Resumo}

Pasquetti, Eduardo; Gonçalves, Paulo B.. Estabilidade estática e dinâmica de torres estaiadas. Rio de Janeiro, 2003. 99p. Dissertação de Mestrado Departamento de Engenharia Civil, Pontifícia Universidade Católica do Rio de Janeiro.

Torres estaiadas são, em geral, estruturas bastante leves e esbeltas e que apresentam comportamento eminentemente não-linear. Assim, a análise de sua estabilidade sob cargas estáticas e dinâmicas é essencial para se ter um projeto econômico e seguro. Neste trabalho estuda-se a estabilidade estática e dinâmica de um modelo plano de torre estaiada. Especial atenção é dada à modelagem dos estais, que são modelados como elementos de mola (linear ou não-linear) ou como cabos inextensíveis. Faz-se, com base no princípio da energia potencial mínima, um estudo da carga crítica e do caminho pós-crítico. Um estudo paramétrico minucioso permite analisar a influência dos diversos parâmetros físicos e geométricos na estabilidade da torre e chegar às melhores configurações para os estais. Na análise dinâmica atenção especial é dada ao estudo paramétrico da freqüência natural. Com base nos resultados da análise estática, analisa-se também o comportamento global da torre em vibração livre e forçada. Em virtude das não linearidades, verifica-se que a torre pode apresentar diversos comportamentos típicos de sistemas não-lineares tais como saltos, bifurcações de período e caos.

\section{Palavras-chave}

Instabilidade; torres estaiadas; cabos, dinâmica não-linear 


\section{Abstract}

Pasquetti, Eduardo; Gonçalves, Paulo B.. Static and dynamic stability of guyed towers. Rio de Janeiro, 2003. 99p. MSc Dissertation - Department of Civil Engineering, Pontifícia Universidade Católica do Rio de Janeiro.

Guyed towers are in general very light and slender structures and their behavior under static and dynamic loads is eminently nonlinear. Thus the analysis of its stability under static and dynamic loads is an essential step in obtaining an economic and safe project. In this work the static and dynamic stability analysis of a plain model of a guyed tower is studied. Special attention is given to the modeling of the stays; here they are modeled as spring elements (linear or nonlinear) or as inextensible cables. A study of the critical load and the postcritical nonlinear equilibrium paths is conducted based on the principle of the minimum potential energy A detailed parametric analysis is performed to identify the influence of the physical and geometric parameters of the system on the stability of the tower. This allows one to choose the best configurations for the stays. In the dynamic analysis special attention is given to the parametric study of the system natural frequencies. Based on the results of the static analysis, the global behavior of the tower under free and forced vibration is also analyzed. Due to the inherent nonlinearities, the tower may present dynamic responses typical of nonlinear systems such as jumps, period bifurcations and chaos.

\section{Keywords}

Instability; guyed towers; cables, nonlinear dynamics 


\section{Sumário}

1 Introdução 19

1.1. Motivação 23

1.2. Objetivos 23

1.3. Organização do trabalho 23

2 Cabos em catenária $\quad 24$

2.1. Determinação das reações sobre os pontos de fixação do cabo 24

2.2. Determinação da inclinação inicial da catenária incompleta- $\alpha_{0} \quad 26$

2.3. Determinação do comprimento do cabo e sua projeção horizontal em catenárias completas 28

2.4. Equação da catenária 28

2.5. Variação da forca de tração ao longo do cabo 29

2.6. Deformações dos cabos 30

2.7. Principais características dos cabos de aço 32

3 Análise da Estabilidade - Modelo de Molas 34

3.1. Modelo de molas lineares 34

3.1.1. Energia Potencial total 35

3.1.2. Energia interna de deformação 35

3.1.3. Trabalho das forças externas 36

3.1.4. Equação do caminho pós-crítico de equilíbrio 37

3.1.5. Análise da carga crítica 38

3.1.5.1. Valor limite do parâmetro de protensão $\xi_{0} \quad 40$

3.1.5.2. Análise da carga crítica a partir da equação na forma dimensional 41

3.1.5.3. Consideração de várias cargas axiais 42

3.1.6. Caminho pós-crítico de equilíbrio 42

3.1.6.1. Análise da estabilidade do caminho pós-crítico 44

3.1.7. Modelos Bi-lineares 46

3.1.8. Caminhos de equilíbrio considerando imperfeições iniciais 49

3.1.8.1. Influência da imperfeição geométrica - $\theta_{0} \quad 50$

3.1.8.2. Influência da excentricidade do carregamento - $e_{1}$ 
3.1.8.3. Influência da carga lateral - q

3.1.9. Influência do aumento no número de estais 52

3.1.9.1. Análise da carga crítica de um modelo de 4 molas 53

3.1.9.2. Análise da estabilidade do caminho pós-crítico de um modelo com 4 molas

3.2. Modelo de molas não lineares $\quad 55$

3.2.1. Energia interna de deformação 55

3.2.2. Equação do caminho pós-crítico de equilíbrio 56

3.2.3. Análise da carga crítica 56

3.2.3.1. Variação da carga crítica em relação as constantes de mola 56

3.2.3.2. Variação da carga crítica em relação a inclinação da mola 57

3.2.3.2.1. Influência de $\alpha_{1}$ e $\gamma_{1}$

3.2.3.3. Variação da carga crítica em relação ao alongamento inicial da mola 58

3.2.4. Análise da estabilidade do caminho pós-crítico de equilíbrio 60

3.2.5. Caminhos de equilíbrio considerando imperfeições iniciais 63

3.2.6. Influência de um número maior de molas 64

4 Análise da Estabilidade - Modelo de Cabos 65

4.1.1. Variação da energia devida a mudança de configuração dos cabos 65

4.1.2. Equação do caminho pós-crítico de equilíbrio 66

$\begin{array}{ll}\text { 4.1.3. Análise da carga crítica } & 67\end{array}$

4.1.3.1. Comprimento do cabo constante 67

4.1.3.2. Influência do comprimento do cabo na carga crítica 68

4.1.3.3. Influência do peso e comprimento do cabo 69

4.1.3.4. Influência do fator de protensão f 70

4.1.4. Análise do caminho pós-crítico de equilíbrio 71

4.1.4.1. Estabilidade do caminho pós-crítico 72

4.1.4.2. Afrouxamento de cabos e estabilidade $\quad 73$

4.1.5. Caminhos de equilíbrio considerando imperfeições iniciais 74

4.2. Representação de cabos por molas não-lineares 76

4.2.1. Funções que representam a variação da força do cabo 76

5 Análise Dinâmica da Estabilidade $\quad 79$

5.1. Critério dinâmico da estabilidade 79

5.2. Energia Cinética $\quad 79$

5.3. Equação do movimento 
5.3.1. Modelo de molas $\quad 81$

5.3.2. Modelo de cabos 81

5.4. Freqüência natural 82

5.4.1. Máxima freqüência natural $\quad 84$

5.5. Solução numérica da equação de movimento 85

5.6. Amortecimento crítico 86

5.7. Análise da estabilidade e natureza do movimento 86

5.7.1. Análise do sistema autônomo 87

5.7.2. Análise do sistema não autônomo 91

5.7.2.1. Bifurcação por dobramento de período 92

6 Conclusões 96

$\begin{array}{ll}\text { 6.1. Sugestões para continuidade desta pesquisa } & 97\end{array}$

7 Referências Bibliográficas $\quad 98$ 


\section{Lista de figuras}

Figura 1.1: Modelo de uma torre estaiada. $\quad 19$

Figura 1.2: Torres estaiadas com seção triangular. $\quad 20$

Figura 1.3: Torres estaiadas com seção tubular. 20

Figura 1.4: Configurações usuais de torres estaiadas. 21

Figura 2.5: (a) Algumas configurações de cabos; (b) diagrama de corpo livre de um cabo. 24

Figura 2.6: cabo em uma configuração frouxa. $\quad 27$

Figura 2.7: (a) Variação da tração ao longo do cabo; (b) forma da catenária 30

Figura 2.8: Nomenclatura empregada na fabricação de cabos de aço, figura retirada da referência [12]. $\quad 32$

Figura 2.9: Tabela de cabos para torres estaiadas, referência [12]. 33

Figura 3.10: Modelo de molas: (a) configuração inicial. (b) configuração perturbada. 34

Figura 3.11: Variação do parâmetro de carga crítica em função dos parâmetros adimensionais. 39

Figura 3.12: Caminho pós-crítico para diferentes valores da inclinação da mola.

Figura 3.13: Derivada da curvatura inicial com relação a $\xi_{0}$ e $\xi_{1}$. $\quad 45$

Figura 3.14: caminhos pós-críticos na fronteira de estabilidade. 46

Figura 3.15: Torre com apenas uma mola $\quad 47$

Figura 3.16: Caminhos de equilíbrio para o modelo com apenas uma mola e $\begin{array}{ll}\text { duas molas. } & 47\end{array}$

Figura 3.17: Reações das molas sobre a torre e seus respectivos braços de alavanca. $\quad 48$

Figura 3.18: Caminhos de equilíbrio considerando que as molas resistem somente a esforços de tração. $\quad 48$

Figura 3.19: Rompimento de estais: (a) rompe-se a mola tracionada. (b) rompese a mola comprimida. $\quad 49$

$\begin{array}{lr}\text { Figura 3.20: Modelo imperfeito } & 49\end{array}$

Figura 3.21: Caminho de equilíbrio do modelo perfeito e com dois níveis de imperfeição inicial. $\quad 50$

Figura 3.22: Caminhos de equilíbrio do modelo perfeito e com dois níveis de excentricidade. 
Figura 3.23: Caminho de equilíbrio do modelo perfeito e com dois níveis de carregamento lateral. 52

Figura 3.24: Modelo com 4 molas. 52

Figura 3.25: Variação da carga crítica: (a) em função da inclinação das molas e (b) em função das constantes de mola. 53

Figura 3.26: Caminhos pós-críticos para diferentes inclinações das molas. $\quad 54$

Figura 3.27: variação da carga da carga crítica em função do deslocamento inicial para dois valores de $\gamma_{1}$.

Figura 3.28: Caminhos pós-críticos de equilíbrio para diferentes valores de $\alpha_{1}$ e $\Delta I_{01}$

Figura 3.29: Variação $d^{2} \Delta \pi / d \theta^{2}$ : (a) em função de $k_{1}$ e $\alpha_{1}$ na ausência de prétensionamento; (b) em função de $k_{2}$ e $\alpha_{1}$ na ausência de pré tensionamento. (c) e (d) os casos anteriores com pré-tensionamento. 61

Figura 3.30: Caminhos pós-criticos de equilíbrio.

Figura 3.31: Caminhos de equilíbrio: (a) imperfeições iniciais; (b) excentricidade do carregamento.

Figura 3.32: Caminhos de equilíbrio com a presença de carregamento lateral. 63

Figura 4.33: Torre estaiada. Modelo com cabos inextensíveis: (a) configuração fundamental de equilíbrio; (b) configuração perturbada (perturbação rotação $\theta$ ).

Figura 4.34: Inclinação e projeções da reta que une os pontos de fixação do cabo.

Figura 4.35: Variação da carga crítica em função da inclinação $\alpha$.

Figura 4.36: Variação da carga crítica em função da inclinação e do comprimento do cabo.

Figura 4.37: (a) Variação da carga crítica em função do peso do cabo. (b) variação da carga crítica em função do comprimento cabo. 70

Figura 4.38: Variação da carga crítica em função do pré-tensionamento. $\quad 70$

Figura 4.39: Caminhos pós-críticos de equilíbrio para diferentes inclinações. 71

Figura 4.40: Curvas limite para o qual o sistema apresenta caminhos pós-críticos estáveis com carga crítica positiva.

Figura 4.41 (a-f) Caminhos de equilíbrio considerando dois ou somente um cabo.

(g) análise qualitativa do equilíbrio. $\quad 74$

Figura 4.42: Modelo imperfeito.

Figura 4.43: modelo com cabos com comprimentos diferentes 75 
Figura 4.44: Comparação entre a força dada pela catenária e a equação (4.8) com as constantes determinadas pelo método dos mínimos quadrados. 78

Figura 5.45: Posição de um elemento da barra de comprimento infinitesimal, $d s$.

Figura 5.46: Variação da freqüência natural circular: (a) em função de $\alpha$. (b) em função de $\lambda$.

Figura 5.47: Variação da freqüência natural circular: (a) em função do parâmetro de pré-tensionamento, (b) em função do parâmetro de geometria.

Figura 5.48: Variação do quadrado da freqüência natural circular em função do parâmetro de carga.

Figura 5.49: Variação da Freqüência natural em função de $\alpha$.

Figura 5.50: Vibração livre: (a) comparação entre o modelo de molas lineares e o modelo de molas não lineares; (b) comparação entre o modelo de cabos e o modelo de molas usado para representar cabos. 85

Figura 5.51: Relação entre a superfície de energia e o plano fase. 87

Figura 5.52: Caminho pós-crítico. $\quad 88$

Figura 5.53: Parâmetro de carga menor que o crítico: (a) Superfície de energia (b) plano fase.

Figura 5.54 Plano fase para um valor de $\lambda$ levemente maior que o crítico.

89

Figura 5.55: Caminho pós-crítico e parâmetro de carga utilizado em cada caso.

Figura 5.56 Superfície de energia para carregamento inferior ao crítico 90

Figura 5.57: Parâmetro de carga maior que o crítico: (a) Superfície de energia (b) plano fase.

Figura 5.58: Plano fase para diversos níveis de carga lateral, com $\lambda$ maior que $\lambda$ crítico.

Figura 5.59: Plano fase para diversos níveis de carga lateral, com $\lambda$ menor que $\lambda$ crítico.

Figura 5.60: Diagrama de bifurcação do sistema não-autônomo para valores crescentes da amplitude do carregamento harmônico. Variação da coordenada generalizada $\theta$ da seção de Poincaré em função do parâmetro $q$.

Figura 5.61: Plano fase e mapeamento de Poincaré para diferentes carregamentos extraídos da Figura 5.16. 


\section{Lista de tabelas}

Tabela 2.1: Deformação do cabo em função da inclinação da reta que passa

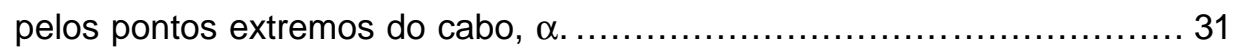




\section{Lista de símbolos}

A, ponto de fixação do cabo ao chão; área da seção transversal da barra;

$A_{m}$, área metálica da seção transversal do cabo;

A', ponto de fixação do cabo ao chão em catenárias completas;

$B$, ponto de fixação do cabo àtorre;

$E$, módulo de elasticidade longitudinal do cabo;

$F, \quad$ força que age em uma mola;

$F_{0}$, força de pré-tensionamento da mola;

$H$, distância entre o ponto de aplicação da carga axial e o apoio da barra;

L, comprimento da barra; função de Lagrange;

$P, \quad$ carga axial; ponto no plano de Poincaré;

$P_{c r i}, \quad$ carga axial crítica;

$T$ força do cabo, energia cinética;

$T_{q}$, período do carregamento;

$T_{x}, T_{x}(\theta)$, componente horizontal da força que o cabo exerce sobre a barra;

$T_{y}, T_{y}(\theta)$, componente vertical da força que o cabo exerce sobre a barra;

$W$ trabalho das cargas externas;

a, coeficientes do polinômio obtido pelo método dos mínimos quadrados;

b, curvatura inicial do caminho pós-crítico;

c, coeficiente de amortecimento;

$c_{c r i}$, coeficiente de amortecimento crítico;

$d \Delta U$, derivada da energia interna de deformação em relação a $\theta$;

$d x$, comprimento infinitesimal segundo a direção $x$; derivada da função que define a posição do ponto $B$ do cabo, segundo a direção $x$, em relação ao ponto A;

ds, comprimento infinitesimal medido ao longo do cabo; 
$d y$, comprimento infinitesimal segundo a direção $y$; derivada da função que define a posição do ponto $B$ do cabo, segundo a direção $y$, em relação ao ponto $A$;

$f \quad$ fator de protensão; freqüência natural;

$f(\theta) \quad$ força elástica;

$k$, constantes de mola;

$F_{0}$, força de pré-tensionamento;

$h$, distância entre os pontos de fixação da mola àtorre e o apoio da barra;

$k_{e q}$, rigidez equivalente;

$k_{e q l}$, rigidez equivalente linearizada;

$m_{e q}$, massa equivalente;

nc, número de cabos;

$n m$, número de molas;

$n p \quad$ número de cargas concentradas;

$p$, peso próprio da barra;

$q$, carga lateral;

$s$, comprimento do cabo;

s', comprimento do cabo em catenária completa;

$s_{x}$, projeção horizontal do cabo;

$s_{x}{ }^{\prime}$, projeção horizontal do cabo em catenária completa;

$s_{y}$, projeção vertical do cabo;

$s_{0}$, parte do cabo que fica sobre o chão, em catenárias completas;

$x$, eixo na direção horizontal; função posição da barra;

$v$, velocidade

$y$, eixo na direção vertical; equação que define a posição da catenária segundo o eixo vertical; função posição da barra, posição da carga de peso próprio em relação ao comprimento da barra;

$\bar{y}$, centro de gravidade da barra;

$w$, peso do cabo $(\mathrm{N} / \mathrm{m})$

$\Delta U$, variação da energia interna de deformação;

$\Delta W$, variação do trabalho das cargas externas; 
$\Delta /, \quad$ alongamento do cabo, mola;

$\Delta I_{0}$, alongamento inicial da mola não linear;

$\Delta J_{\text {olim k1 }}$, valor limite de $\Delta l_{0}$ para o qual, a derivada da carga crítica em relação a $k_{1}$ é positiva, para o modelo de mola não linear;

$\Delta l_{\text {llim k2 }}$, valor limite de $\Delta l_{0}$ para o qual, a derivada da carga crítica em relação a $k_{2}$ é positiva, para o modelo de mola não linear;

$\Delta J_{\text {olima1k1 }}$ valor limite de $\Delta I_{0}$ para o qual, os termos que contém $k_{1}$ na derivada da carga crítica em relação a inclinação são negativos (modelo de mola não linear);

$\Delta \Delta_{0 \text { lima1k2 }}$ valor limite de $\Delta J_{0}$ para o qual, os termos que contém $k_{2}$ na derivada da carga crítica em relação a inclinação são negativos (modelo de mola não linear);

$\Delta I_{\text {max }}$,alongamento inicial que provoca um máximo para a carga crítica;

$\Delta I_{0 \min }$, alongamento inicial que provoca um mínimo para a carga crítica;

$\Delta I_{\theta}$, alongamento da mola devido a uma rotação $\theta$ da barra;

$\Delta \pi, \quad$ variação da energia potencial total;

$\Delta T$, variação da energia cinética;

$\Gamma$, posição da carga axial em relação ao comprimento da barra;

$\alpha \quad$ inclinação do cabo; inclinação do segmento de reta que passa pelos pontos $\mathrm{A}$ e $\mathrm{B}$;

$\varepsilon$, função erro na resolução da catenária incompleta;

$\gamma$, relaciona a altura de fixação da mola a barra com o comprimento da mesma;

$\gamma_{\min 1}$ valor mínimo que $\gamma$ pode assumir, para que $\Delta I_{0 \max }$ seja diferente de zero;

$\gamma_{\min 2}$ valor mínimo que $\gamma$ pode assumir, para que haja algum ponto em que a derivada da carga crítica em relação a $\Delta / 0_{0}$ seja zero;

$\lambda$, autovalor;

$\lambda_{p}$, parâmetro adimensional de carga;

$\lambda_{\text {pcri }}$, parâmetro adimensional de carga crítica; 
$\theta$, coordenada generalizada que descreve a posição da barra em relação a vertical;

$\dot{\theta}$, velocidade da barra em relação a vertical;

$\rho$, densidade da barra;

$\xi_{p}$, parâmetro adimensional relacionado àcarga de peso próprio;

$\xi_{0}$, parâmetro adimensional relacionado àforça de pré -tensionamento da mola;

$\xi_{1}$, parâmetro adimensional relacionado as constantes de mola;

$\omega, \quad$ freqüência natural circular; 